\title{
Digital integrators for condition monitoring: a DC and multitone signal analysis
}

\author{
L. Peretti \\ Department of Power Technologies \\ ABB Corporate Research \\ Västerås, Sweden \\ luca.peretti@se.abb.com
}

\begin{abstract}
The digital implementation of the integral operator is a delicate part in electrical drives. Many solutions are available in the literature, which avoid the well-known output drift of ideal digital integrators when a spurious DC offset is present at their input. Such solutions try to extend the integration accuracy toward the zero frequency as well, aiming to broaden their usability range for real applications (low-speed region of electrical machines, for example). So far, very few investigations have been performed as regards to the performances of driftfree integrators for cases where the integration is used within estimation and conditioning monitoring algorithms. This paper aims to investigate different known digital offset-free integrals, in terms of their output accuracy with respect to DC and multitone input signals, for a specific application where they could be exploited for condition monitoring. Simulation and experimental results are presented as well.
\end{abstract}

\section{INTRODUCTION}

The frequency analysis of variables measured in an electrical machine is an aspect of rising interest in the drive control scenario, especially when considering condition monitoring and diagnostics. In these applications, when a deviation from the normal behaviour is detected, the monitoring algorithm could trigger warning messages to the operator or initiate counterbalancing control actions [1].

In a typical case, signals such as the phase currents could be used to determine the presence of a failure in the machine, as in [2]. The phase currents are not the only signals available in a conventional drive: phase voltages could be either measured or estimated through the compensation of inverter nonlinearities [3], and may bring information on harmonics when closed-loop controls are employed. The torque could also be interpreted as an indicator of malfunctioning [4]. Vibration signals could also be properly placed on the equipment under test as part of a condition monitoring tool [5].

The frequency analysis is not necessarily performed on raw signals coming from the drive sensors. A typical example is represented by the electromagnetic torque, which could be analysed and estimated through the cross-product between the currents and the flux linkages.

In general, when dealing with raw signals, a prerequisite for a successful harmonic analysis is the selection of a suitable sensor which does not distort the signal in the bandwidth of interest. When the analysed variables are a combination of raw and estimated signals, as the electromechanical torque case where the currents are measured and the flux linkages must be estimated, it is also essential that the models used for the estimation preserve the original harmonic information without amplitude and/or phase distortion.

Extensive literature has been produced as regards to flux linkage estimation, a major distinction being closed-loop observers of open-loop estimators. In the first case, the electrical machine parameters are used to implement closed-loop observers. These observers are usually exploited for more purposes rather than the flux linkage estimation only. Examples are found in [6], [7], [8], [9], [10], [11], [12], [13], [14].

Open-loop estimators, instead, calculate the flux linkages as the integral of the back-electromotive force. In this case, the integrators are not embedded in closed-loop observers and do not require, in principle, any tuning. The only required machine parameter for the calculation of the back-electromotive force is the stator resistance, while other parameters as the machine inductances do not affect the result of the estimation, as it happens in closed-loop estimators. However, the biggest issue of open-loop estimators is their output drift in presence of spurious DC signals at their input, which are unavoidable in modern digital electrical drives. Extensive research have been performed in the field of drift-free open-loop estimators, as reported in [1], [15], [16], [17], [18], [19], [20], [21].

When considering a frequency analysis for condition monitoring purposes, drift-free open-loop integrators might be considered for their potential lower parameter sensitivity. However, very few investigation has been made as regards to the evaluation of the integrators in terms of DC signal rejection (transient required to obtain an offset-free signal in output) and the capability of performing a correct integration in presence of two (or more) frequencies at once.

This paper investigates the behaviour of different drift-free open-loop integrators, based on existing solutions found in the literature and briefly described in Sect. II. By setting some requirements for the application of interest in Sect. III, the integrators will be tuned according to the DC-input rejection test described in Sect. IV. Then, each integrator performance to multitone input signals, described in Sect. V-A, will be evaluated in Sect. V-B and Sect. V-C. Experimental evidence is included in Sect. VI, showing the existence of integration mismatches that may impact condition monitoring and diagnostics applications. 


\section{OVERVIEW OF DIGITAL INTEGRATORS}

This section describes the different algorithms for offset-free integration of the back-electromotive force, for flux linkage estimation and thus torque estimation in electrical drives, listed in chronological order as they have been found in the literature. Each of the solution is briefly analysed.

\section{A. Cascade of low-pass filters with varying time constant}

This algorithm, one of the first in the field, was proposed in [15]. The integrator is decomposed in a cascade of low-pass filters, the number of which can be varied but once selected, it will be fixed during real-time operation. The low-pass filters are equal to each other, and their time constant is the inverse of the actual electrical speed. In overall, the cascade of lowpass filters must have a magnitude and phase shift equal to that of an ideal integrator only for the measured electrical speed. To this purpose, [15] establishes two equations that must be fulfilled for any change of electrical speed, resulting in an online update of a correction gain and of the filters time constant.

\section{B. Offset cancellation by means of scalar product}

The solution in [16] considers that the input and the output signals of an integrator should always be shifted by $\pi / 2$ radians with respect to each other, and therefore the scalar product between them should always be equal to zero.

As a first step, the integrator $1 / s$ is divided into two transfer functions $1 /\left(s+\omega_{c}\right)$ and $\omega_{c} /\left(s+\omega_{c}\right)$ rearranged in a sum and a loop, where $\omega_{c}$ is an angular frequency selected by the designer. As a second step, a cartesian-topolar transformation calculates the amplitude and the phase of the flux linkage vector, which is the output of the integrator. The amplitude of the flux linkage is used to normalize an error signal, which is obtained as the scalar product between the flux linkage components and the back-electromotive force components in the $\alpha \beta$ reference frame. A proportional-integral regulator tries to force the normalised error to zero, its output being transformed back in cartesian coordinates using the flux linkage angle. This component component is then processed as input of the transfer function $\omega_{c} /\left(s+\omega_{c}\right)$, closing the loop.

\section{Low-pass filter with changing time constant}

The solution in [17] is not very different from the one in [15] in its concept: it is a low-pass filter (only one, in this case), whose time constant is varied with respect to the value of the electrical speed. A gain compensator and a phase adjustment block are present as well. The main difference is that the time constant is calculated as a multiple (by a factor of $k$ ) of the inverse of the electrical speed, while in [15] the time constant of the filter is exactly the inverse of the electrical speed. The electrical speed is calculated and fed back from the output of the integrators.

Another difference with respect to [15] is the vectorial nature of the solution. This has no real impact on the integration itself, except in the phase compensation block. In [15], the cascade of the filters was designed in such a way that the overall phase shift of the output would be equal to $-\pi / 2$ radians. In [17], the required phase shift is calculated and used for a vectorial rotation, which shifts the output of the required amount to obtain the overall $-\pi / 2$ radians lag.

\section{Vector-based low-pass filter with changing time constant}

The solution presented in [18] gives to the integration operation a complete vectorial perspective - for many aspects, it can be considered a generalisation of the solution in [17], with an easier implementation. A pure integrator is preceded by an high-pass filter which removes the DC components of the incoming signals. A vectorial approach is used. As in [17], the solution in [18] also calculates the electrical speed from the output signal of the integrator, feeding back the result to the blocks that require it.

\section{E. Offset calculator based on vector distortion in $\alpha \beta$}

The solution in [19] starts by describing a condition in which a pure integration is performed, and the flux linkage in either the $\alpha$ or $\beta$ axis drifts to infinite (positive or negative values).

Then, a limitation of the flux linkage is introduced, justified by considering that in electrical machines there will always be a maximum absolute value of the flux linkage. Any time the drift will incur in the limitation, there will be a difference between the maximum peak and the minimum peak of the saturated flux linkage calculated over one electrical period in the $\alpha \beta$ stationary frame.

By computing the average between the maximum peak and the minimum peak for both the $\alpha$ and $\beta$ axis, and indication of the offset vector direction is given. This direction is filtered and fed back to the input of the pure integrator, in addition to the normal input signal. The overall effect is the offset elimination from the back-electromotive force.

\section{F. High-pass filtering instantaneous compensation}

The solution proposed in [20] combines high-pass filters and pure integrators. The high-pass filters are first applied to the $\alpha \beta$ input components. The input and the output of the highpass filters are routed to a compensation block that calculates the magnitude and the phase shift of the filters transfer function at each time instant. The calculation is vectorial.

The calculated magnitude is used to correct the output of the pure integration which follows the high-pass filter. The phase shift is used for a rotation of the vector obtained from the pure integration, in order to re-establish the $-\pi / 2$ radians shift condition.

\section{G. Low-pass filter with changing time constant and PLL for angle estimation}

The solution presented in [21], which has also been mentioned and used in [1], is a modification of the one in [17]. The main integration block remains the same, being composed by a low-pass filter with a time constant that is function of the electrical speed. In this case, however, the electrical speed is obtained with a phase-locked loop (PLL) acting on the integrator output signals, rather than directly from the components of the output vector as in [17]. 


\section{REQUIREMENTS OF THE APPLICATION}

In the following sections, the integrators described in Sect. II are evaluated with respect to their ability of rejecting a DC input component and performing a correct flux linkage estimation in presence of multitone signals. Since the performances of the integrators vary depending on their tuning, a set of requirements is necessary before starting the evaluation.

Rather than the focus on the correct estimation for frequencies very close to zero, which is a typical requirement in model-based sensorless control algorithms [3], here the interest is towards applications where the presence of multitone signals is quite common. The selected application is the control of wind turbine generators, either synchronous or asynchronous ones, by means of electrical drives.

In this case, the generator is part of a system where many different components contribute to the presence of harmonics in measurable signals. Apart from the generator itself, harmonics could be caused by effects like wind shear and tower shadow [22], tower oscillations [23], gearbox vibrations [5], misalignments of the drive train shaft [4], and many others. Overall, the consideration that the possible harmonics in the measurable signals are found at the fundamental electrical frequency of rotation or its multiples is a simplification of the problem. An example is represented by the resonance effects of a wind turbine tower, as documented in [24]. In this case, the wind gusts hitting the tower lead to temporary oscillations with a main frequency equal to the dominating mechanical resonance of the tower structure. Such oscillations are visible in the torque exerted by the wind on the wind blades, because of the relative movement of the tower with respect to the wind pattern, and therefore in the rotating speed as well [24] This resonant frequency is completely independent from the rotating electrical frequency.

Within this context, the work in [25] has been used as a main reference to determine the lower limits for the integrators. In [25], different direct-drive permanent-magnet synchronous generators designs are evaluated in terms of vibrations caused by the cogging torque/torque ripple on the mechanical drive train of the turbine. A lower limitation of $4 \mathrm{rpm}$ is used as a cut-in speed for the torque (and thus power) production. Based on the generator parameters reported in [25], the cut-in frequency corresponds to $4 \mathrm{~Hz}$. In order to slightly release this limitation, and thus make the evaluation process easier for the integrators, a limit of $6 \mathrm{~Hz}$ is set as the minimum frequency of the flux linkage estimation that is expected to be correctly performed by the drive.

\section{THE DC-INPUT REJECTION TEST}

Based on the considerations of Sect. III, the seven integrators described in Sect. II were tuned by considering the effects of a test input signal with the following characteristics:

- it was composed by a pure sinusoid with unity amplitude and frequency of $6 \mathrm{~Hz}$;

- a DC signal of small amplitude $(0.01 \mathrm{~V})$ was superimposed to the sinusoid.
The transient required to stabilise the output of each integrator was analysed and post-processed to extract it length and the residual DC offset present at the output. A subsequent tuning process was performed for each integrator, such that their transient was concluded within one second after the superimposition of the DC component.

The imposition of such condition is explained as follows. The work in [19] reports that the offset drift is mainly a thermal effect that changes the DC offset very slowly, therefore the response time of the rejection method is not critical. Although true, there are other considerations that may lean towards a fast rejection of the DC component. In particular, non-perfect DC offset compensation in current sensors at drive commissioning stage may lead to a drift of the flux linkage estimation from the very beginning of the drive operation. At the same time, all the solutions analysed in this work are not able of completely rejecting the DC offset, as shown later in Fig. 2, acting similarly to a low-pass filter instead of a pure integrator. For this reason, it becomes very important to stabilize the output of the integrators around a spurious DC level as fast as possible: although empirically, it has been observed that the longer the transient, the larger is the spurious DC component that accumulates at the output of the integrators. This may only worse the behaviour of the remaining control structure.

An example of an integrator transient after the tuning process is shown in Fig. 1, taken from the output of the integrator described in [16]. The transient is well concluded within one second.

Fig. 2 reports the amount of DC offset of the integrators output that have been calculated after each integrator was tuned according to the aforementioned considerations. With the exception of two cases (the integrators of [15] and [19]) that are going to be explained separately, the other integrators behave in a similar manner as regards to the spurious DC offset at their output.

As regards to [15], the nature of its solution is such that the low-pass filters have a time constant equal to the inverse of the actual electrical speed. The main consequence is that the DC rejection transient time is not selectable, but rather dependant on the value of the fundamental harmonic under integration. For the case of this test, the 6-Hz main harmonic returns a time constant of $0.0265 \mathrm{~s}$ for each filter, corresponding to a transient time of approximately $0.1 \mathrm{~s}$. Tihs ten-fold faster transient time has a beneficial effect on the amount of spurious DC effect in output, as shown in Fig. 2. The price to pay, however, will be clear in Sect. V, where multitone signals will be employed for the validation of the integrator Bode diagram.

As regards to [19], Fig. 2 shows a relatively high spurious DC component at the end of the transient. It is worth to note that the nature of the solution relies on the determination of the period of the fundamental harmonic for the calculation of the maximum and minimum flux within one period. In other words, this integrator solution waits the fundamental harmonic to conclude its period before updating a new value of the offset estimation, and several fundamental harmonic periods 
are required to conclude the transient. As a matter of fact, the transient length for this solution was brought to $25 \mathrm{~s}$ in this test, with no means of further reduction. As a consequence, the accumulated spurious DC offset value was increased, as shown in Fig. 2.

Tab. I reports the value of the parameters for all integrators tuned with a DC rejection transient of one second, with the exceptions mentioned above. The name of the parameters is the same reported in the corresponding papers, with the same meaning.

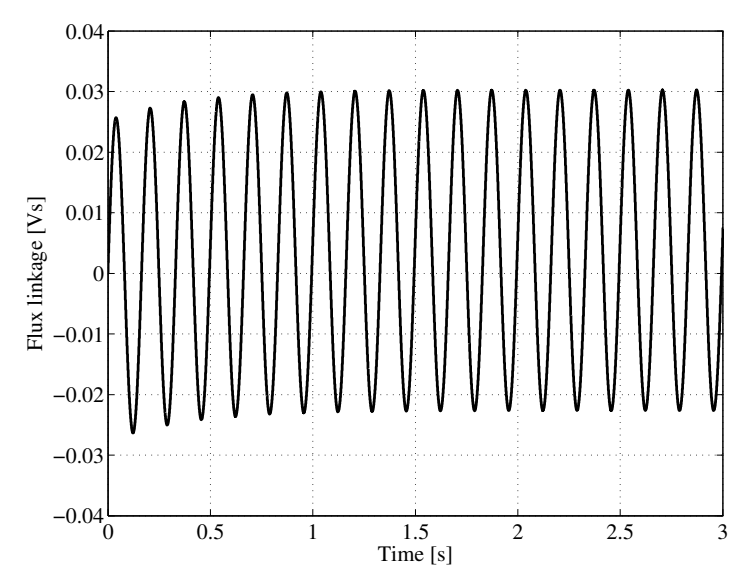

Fig. 1. Example of transient for DC offset rejection concluded within one second, obtained with the integrator [16].

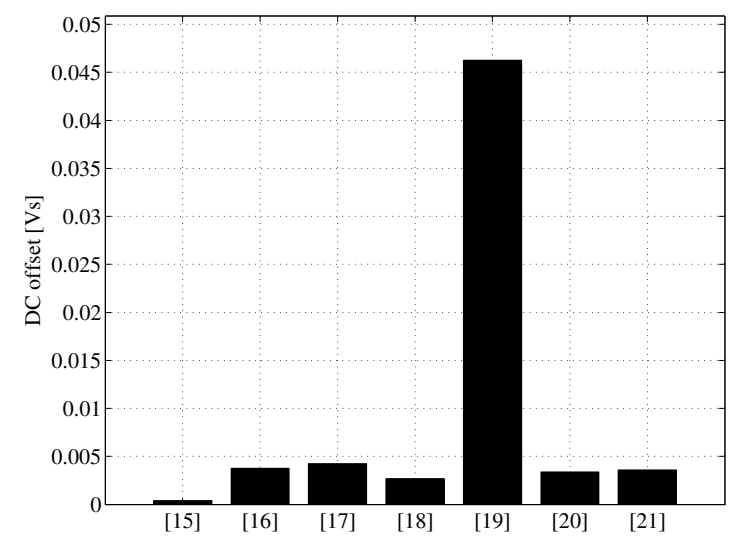

Fig. 2. Residual DC error after the transients for the integrators under evaluation.

Tab. I

PARAMETERS OF THE INTEGRATORS.

\begin{tabular}{c|c|c} 
Integrator solution & Parameter(s) & Value(s) \\
\hline$[15]$ & $n$ & 3 \\
\hline$[16]$ & $\omega_{c}, k_{p}, k_{i}$ & $2 \pi \cdot 0.5,0.01,0.0$ \\
\hline$[17]$ & $k$ & 15 \\
\hline$[18]$ & $\lambda$ & 0.25 \\
\hline$[19]$ & $\lambda_{s}^{*}, \tau_{1}$ & $0.0398,0.0159$ \\
\hline$[20]$ & $\omega_{c}$ & $2 \pi \cdot 0.5$ \\
\hline$[21]$ & $k, k_{p, P L L}, k_{i, P L L}$ & $0.08,1000,200$
\end{tabular}

\section{THE AC-INPUT INTEGRATION TEST}

\section{A. The multitone signals as input}

The evaluation with AC signals was required in order to understand whether the integrators were able to correctly detect multiple harmonics in the input signal. This mode of operation might recall the case where the measured signals are composed by the fundamental harmonic (related to the electrical speed) and other harmonics that carry information on malfunctioning or failures in the electrical machine or the mechanical load.

Signals carrying more than one harmonics at once are known as multitone signals [26]. The construction of a multitone signal is not a difficult task, however one must keep in mind that the sum of many harmonics could lead to signals with high crest factor (ratio between the peak and the RMS value of the signal). As a matter of fact, it is not a good idea to apply high-crest factor signals for system testing, mainly because this could lead to poor signal-to-noise ratio in the processing of the output results. In the worst case, the output signal might not be post-processed by algorithms that try to reconstruct a transfer function. This problem occurs more frequently in experimental tests, but it is a good practice to prevent this situation even in simulation environments, in order to avoid possible numerical errors.

Good multitone signals are created with a special phase relationship between the tones. One of the better solutions is the Newman phase relation, whose mathematical description can be found in [26]. In brief, the Newman phase for multitone signals requires each tone phase to follow the expression:

$$
\varphi_{k}=\frac{\pi(k-1)^{2}}{N}
$$

where $\varphi_{k}$ is the phase of the $k$ th-harmonic (tone), $N$ is the number of generated harmonics (tones) and $k$ is the counter for the $k$-th element. An example of multitone signal with Newman phases containing frequencies from 1 to $20 \mathrm{~Hz}$ (with steps of $1 \mathrm{~Hz}$ ) is shown in Fig. 3a (time domain) and Fig. $3 \mathrm{~b}$ (corresponding FFT). Note that the signal amplitude gets closer to the maximum peak for most of its oscillations, showing a low crest factor. It is easy to prove that the very same multitone signal without the Newman phases has a larger crest factor, with clear consequences on the signal-to-noise ratio of the post-processing results. (a)

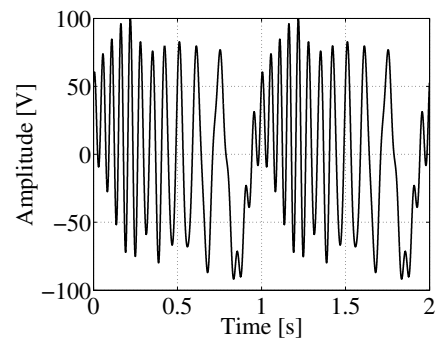

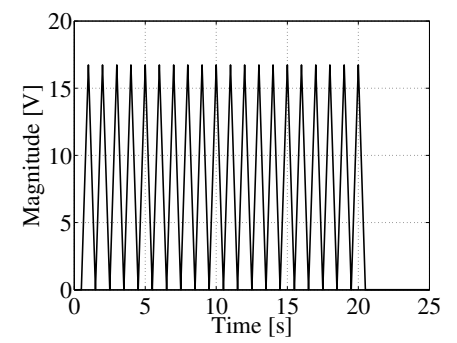

(b)
Fig. 3. Multitone signal with Newman phase: (a) time domain, (b) FFT. 


\section{B. Description of the test}

An input signal resembling a back-electromotive force with many frequencies was created. According to the considerations of Sect. III, the minimum frequency of the input signal was set to $6 \mathrm{~Hz}$, and the maximum was shifted to $1 \mathrm{kHz}$. Steps of $1 \mathrm{~Hz}$ were used in order to cover a good spectrum interval. As for the magnitude of the tones, a more realistic situation was reproduced, where the tones from $7 \mathrm{~Hz}$ onwards had a magnitude 100 times or 10 times lower than then tone at 6 Hz. The results have shown no major differences between the two cases: in this work, only the results with tones 10 times lower than the main harmonic are shown. Note that the input signal has no DC component, since the interest was focused on the integrator performances in presence of multitone signals and the DC rejection test was already performed as described in Sect. IV.

The input signal was fed to all the investigated integrators at the same time, including an ideal digital integrator based on the trapezoidal (Tustin) approximation. Since no DC component was present in the input signal, the Tustin integrator performed a perfect integration that has been used as main reference for the results of all the other integrators.

The simulation was performed for 60 seconds, of which the last 54 seconds have been used for the evaluation of the transfer function of each integrator. Nine windows of six seconds each were extracted from the last 54 seconds, calculating the FFT for each window and each integrator output. The average FFT of the nine windows was taken, and the cross power spectrum between the input and output FFTs was obtained. From the cross power spectrum, an estimation of the transfer function of the integrators was calculated. The sample time of the digital integration was set to $100 \mu \mathrm{s}$. In addition to the transfer functions, the standard deviation error was calculated for each integrator with respect to the transfer function of the ideal integrator, for both the magnitude and the phase.

\section{Results and comments}

The results of the magnitude and phase analysis of the integrators are reported in Fig. 4 and Fig. 6. Fig. 5 and Fig. 7 report the magnitude and phase standard deviation errors for the five best solutions, excluding the worst ones ([15] and [20]) that would make the plots non-readable. Some considerations follow for each integrator under analysis.

Solution [15]: this integrator is with no doubt one of the easiest to implement, but its performances are lacking with respect to the other solutions. Due to the fact that the digital integrator is replicated with low pass filters tuned to fulfil the magnitude/phase requirements for only the main fundamental frequency, all the other harmonics are not correctly reproduced in amplitude and distorted in phase. On the other side, as mentioned in Sect. IV, it is the best solution in terms of DC component rejection. Thus, this solution might be an option only in drives where no spurious harmonics are expected rather than just the main one.
Solution [16]: although showing an interesting linearity in the magnitude Bode diagram (see Fig. 4c), there was a detected phase deviation in the lower region of the spectrum (see Fig. 6c). This behaviour was explained considering the non-linear approach of the solution itself. Specific tests on the same integrator using lower-tone signals, not shown in this work, have shown a tendency of the magnitude to deviate from the ideal integrator towards zero, in a similar way as the phase. Nevertheless, the overall behaviour of the integrator for the application of interest was not bad, although not perfect either.

Solution [17]: there was still a visible sort of linearity in the magnitude Bode diagram (see Fig. 4d), although noisier than [16]. The phase Bode diagram in Fig. 6d was quite noisy. An explanation of this effect might be the automatic calculation of the main electrical frequency from the output of the integrator, required to set the constant in the filter of this solution. A better calculation is probably sought, with the clear consequence of a complication of the scheme.

Solution [18]: it was found to be noisier than [16] and [17], as visible in both Fig. 4e and Fig. 6e. Again, the problem might rely in the self-calculation of the fundamental harmonic of the output signal. By looking at Fig. 5, one might argue that the standard deviation error for the magnitude is not large, being approximately $1.3 \mathrm{~dB}$ for this solution. However, such a value means approximately a $16 \%$ estimation error of the harmonic amplitude. Although probably good enough for closed-loop control applications, such result might be not good enough for condition monitoring solutions, where a precise magnitude estimation is required for a correct prevention of faults.

Solution [19]: it was the best performing one in this test. However, more considerations must be made. On one side, the results in Fig. 4f and Fig. $6 \mathrm{f}$ are not surprising, as the solution does not modify the nature of the integrator itself, rather than changing the input signal in order to make it offset-free. On the other side, this solution has inherent drawbacks as the slowness of the DC rejection transient (see Sect. IV). It was also found that the performances of the algorithm were dependent on a convenient saturation level for the estimated flux linkage. Not last, the algorithm needs to know the fundamental harmonic period in order to estimate the maximum and minimum flux within one period. This is difficult in presence of other harmonics not multiple of the fundamental one. In this work, the knowledge of the fundamental period was given in advance to the algorithm.

Solution [20]: it was found to have a relatively noisy behaviour for higher frequencies (Fig. $4 \mathrm{~g}$ and Fig. 6g). The reason might reside in the instantaneous compensation of the magnitude and phase difference of a high-pass filter with respect of a normal integrator: this might work for a single harmonic, but probably not as well in presence of multiple harmonics.

Solution [21]: as expected, the performances are very similar to [17], and smoothed out because of the presence of the PLL for the estimation of the main fundamental harmonic. The magnitude behaviour is better than the phase, as documented in Fig. 4h and Fig. $6 \mathrm{~h}$. 


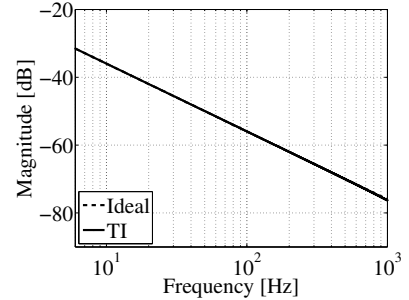

(a)

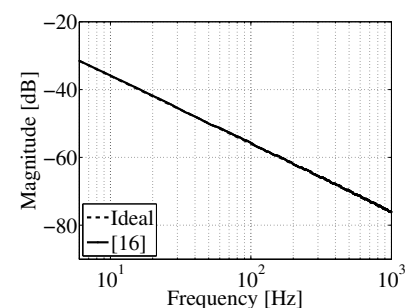

(c)

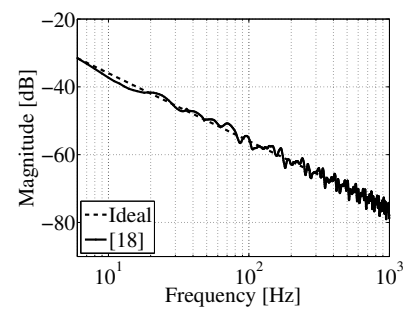

(e)

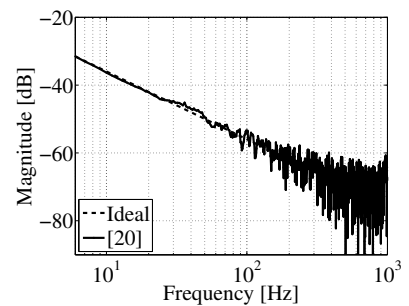

(g)

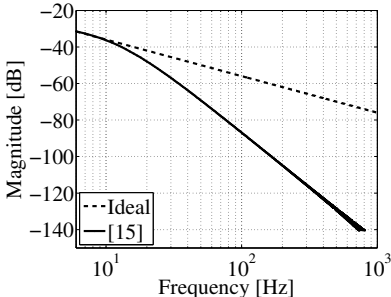

(b)

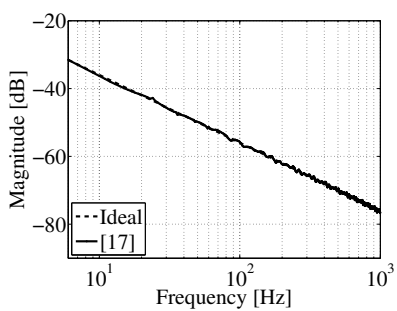

(d)

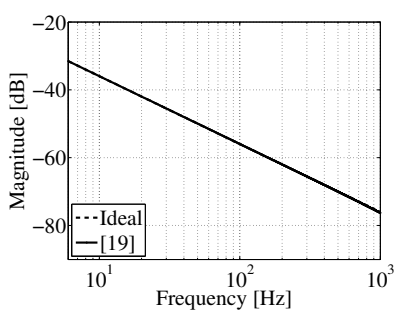

(f)

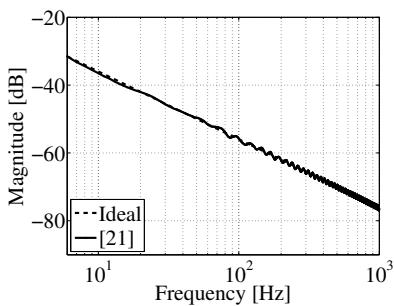

(h)

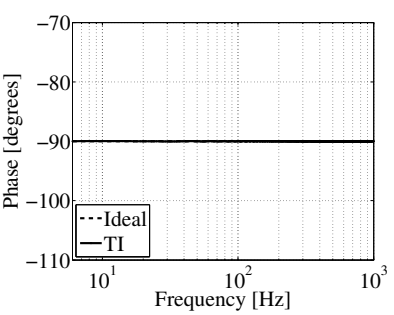

(a)

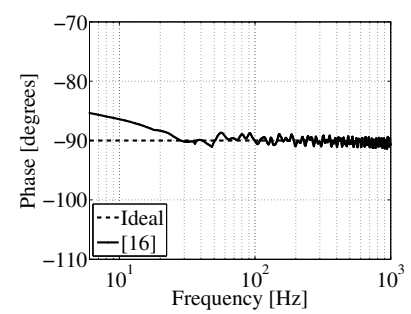

(c)

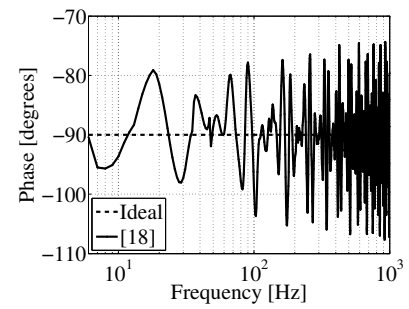

(e)

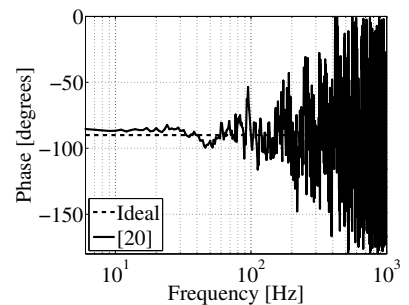

(g)

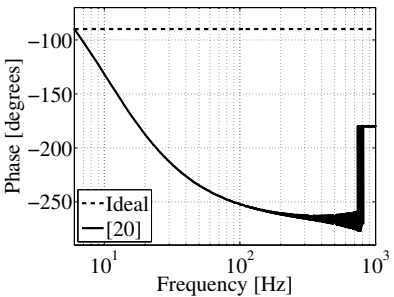

(b)

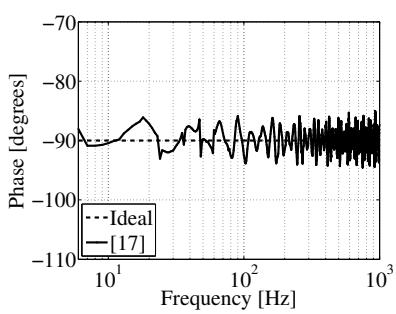

(d)

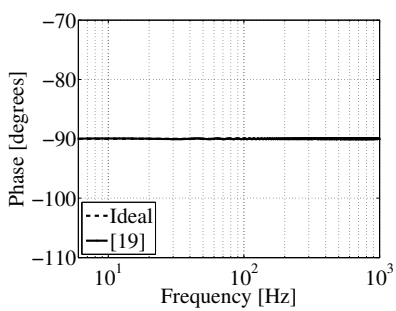

(f)

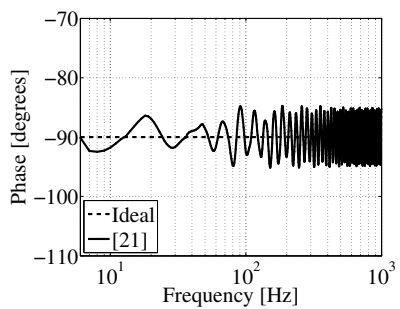

(h)

Fig. 4. Simulation results, magnitude: (a) Tustin integrator, (b) [15], (c) [16] (d) [17], (e) [18], (f) [19], (g) [20], (h) [21]

Fig. 6. TSimulation results, phase: (a) Tus
(d) [17], (e) [18], (f) [19], (g) [20], (h) [21]

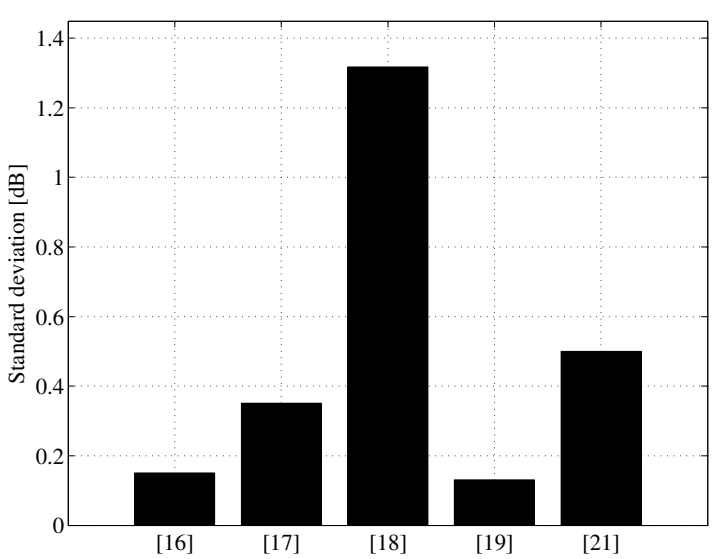

Fig. 5. Magnitude standard deviation error for the best five integrators.

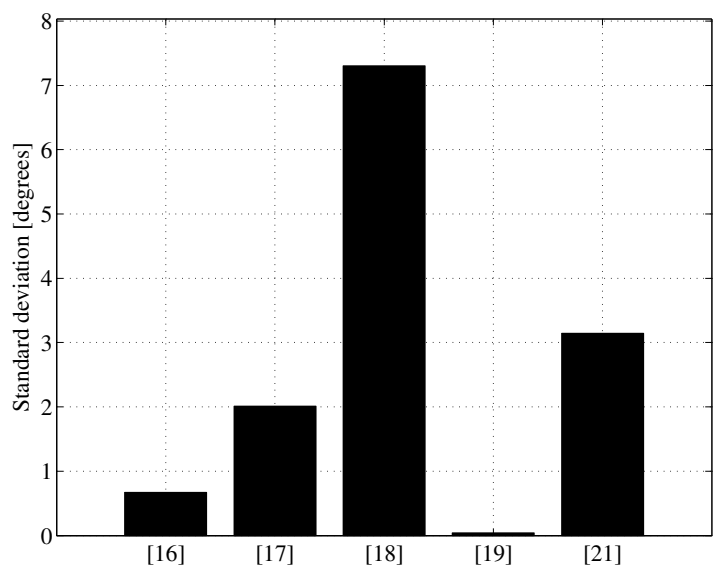

Fig. 7. Phase standard deviation error for the best five integrators. 


\section{EXPERIMENTAL RESULTS}

A set of experimental tests were performed in laboratory premises to validate the simulations of Sect. V. The test bench was composed by two $2-\mathrm{kW}$ synchronous machines (surface permanent magnets) in a back-to-back connection, each controlled with a variable speed drive (ABB ACSM1). One drive was controlled through a custom control board mounting a Xilinx Virtex 6 FPGA, where the algorithms have been developed. A picture of the setup is shown in Fig. 11.

In order to reproduce some frequencies of the spectrum analysed in Sect. V, one of the two machines was controlled with a simple open-loop algorithm where the main voltage vector was rotating at $6 \mathrm{~Hz}$, as the minimum frequency requested in Sect. III. On the top of the reference voltage vector, voltage harmonics were injected at the following frequencies: $15 \mathrm{~Hz}$, $40 \mathrm{~Hz}, 70 \mathrm{~Hz}, 168 \mathrm{~Hz}, 503 \mathrm{~Hz}$ and $794 \mathrm{~Hz}$.

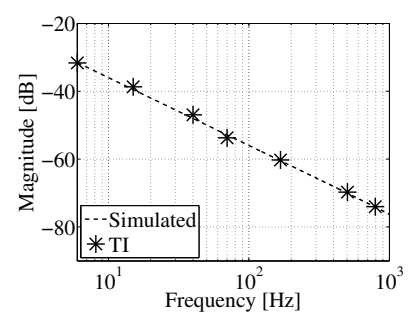

(a)

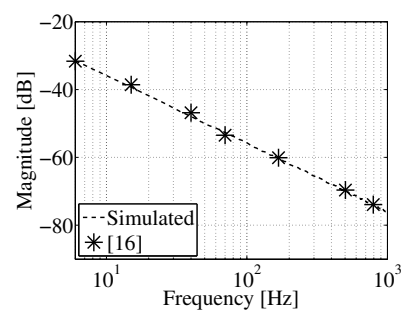

(c)

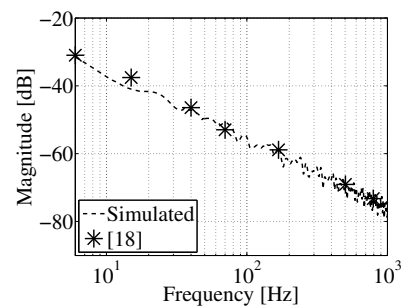

(e)

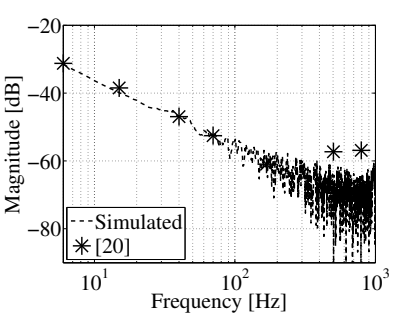

(g)

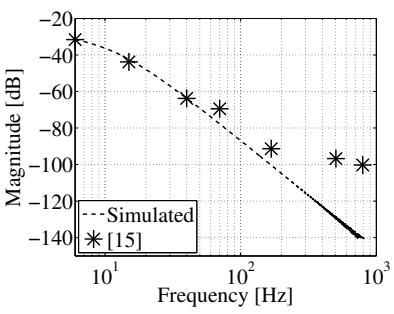

(b)

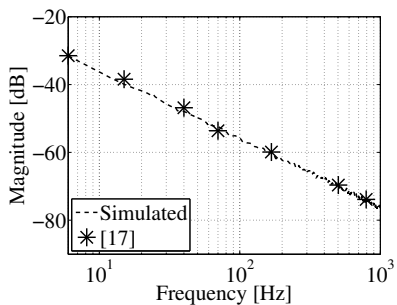

(d)

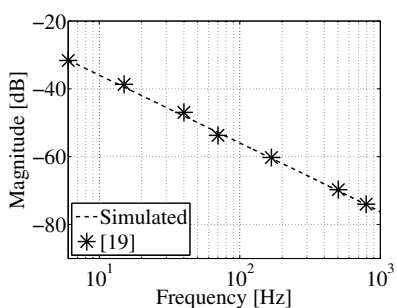

(f)

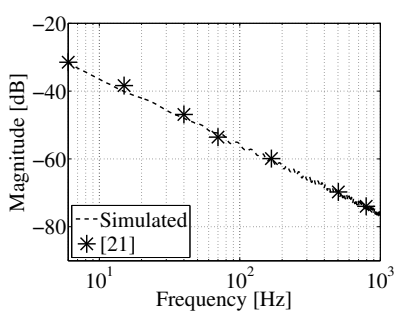

(h)

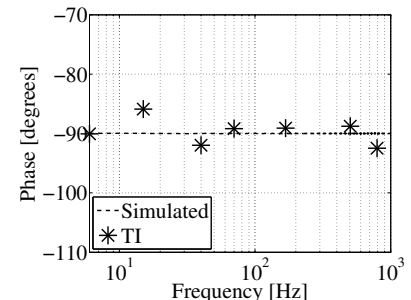

(a)

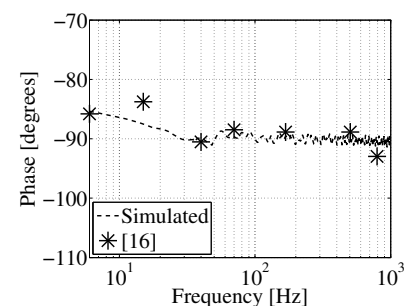

(c)

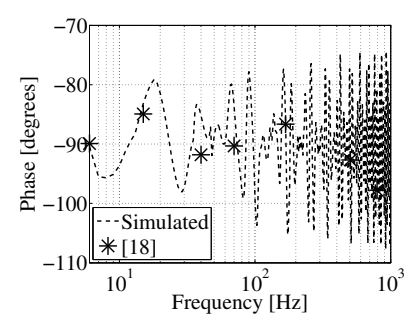

(e)

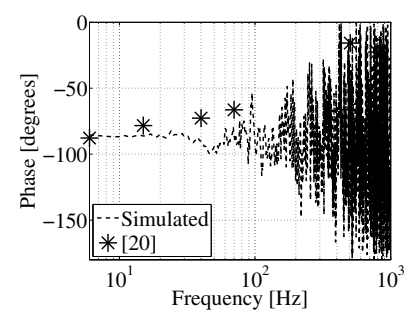

(g)

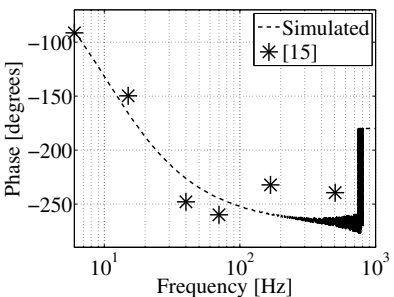

(b)

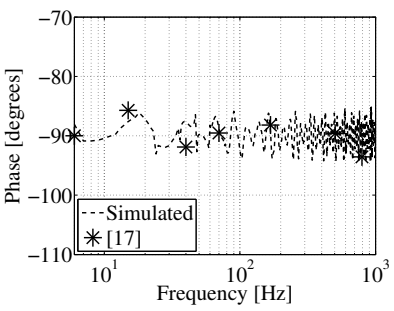

(d)

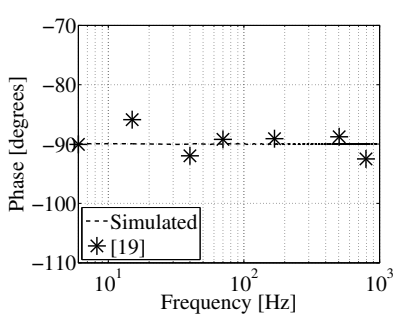

(f)

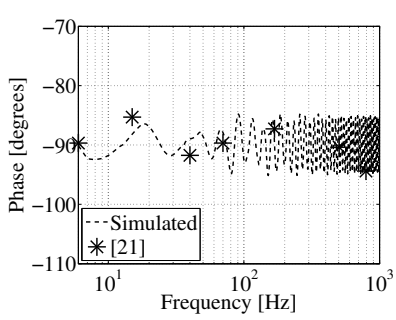

(h)
Fig. 8. Experimental results, magnitude: (a) Tustin integrator, (b) [15], (c) [16], (d) [17], (e) [18], (f) [19], (g) [20], (h) [21].
Fig. 9. Experimental results, phase: (a) Tustin integrator, (b) [15], (c) [16], (d) [17], (e) [18], (f) [19], (g) [20], (h) [21] 


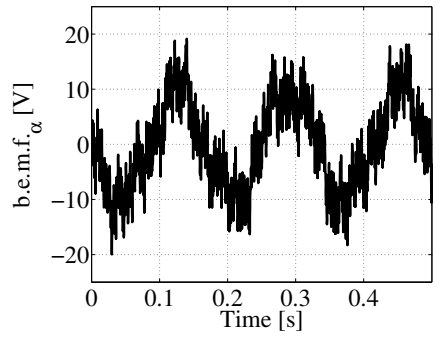

(a)

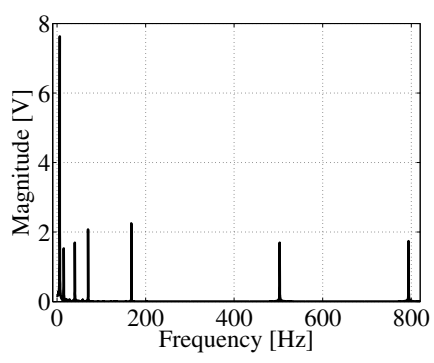

(b)
Fig. 10. Back-electromotive force: (a) time domain, (b) FFT.

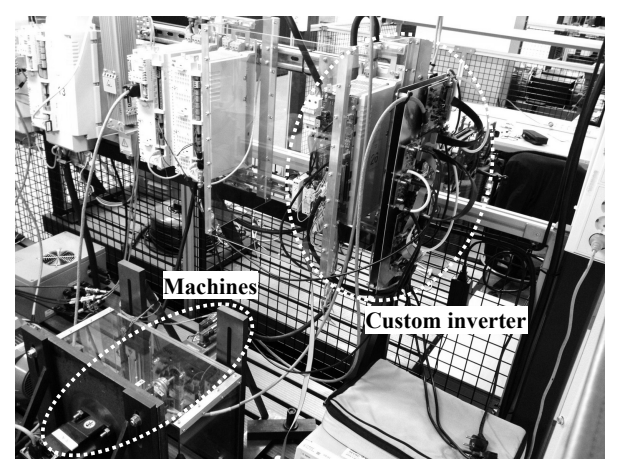

Fig. 11. The experimental test bench.

\section{CONCLUSIONS}

Different algorithms for drift-free open-loop digital integration of the back-electromotive force in electrical machines were analysed. Their performances were evaluated in presence of DC components and multitone signals at their input, recording their deviation with respect to the ideal integrator case. The simulation and experimental analysis showed that some integrator solutions may deviate from the ideal behaviour. This result must be taken into account when performing harmonic analysis of estimated flux linkages or torque, if the focus is on condition monitoring and diagnostics applications.

\section{REFERENCES}

[1] S. H. Kia, H. Henao, and G.-A. Capolino, "Torsional vibration assessment using induction machine electromagnetic torque estimation," IEEE Trans. Ind. Electron., vol. 57, no. 1, pp. 209-219, Jan. 2010.

[2] W. L. Roux, R. G. Harley, and T. G. Habetler, "Detecting rotor faults in low power permanent magnet synchronous machines," IEEE Trans. Power Electron., vol. 22, no. 1, pp. 322-328, Jan. 2007.

[3] J. Holtz and J. Quan, "Sensorless vector control of induction motors at very low speed using a nonlinear inverter model and parameter identification," IEEE Trans. Ind. Appl., vol. 38, no. 4, pp. 1087-1095, Jul./Aug. 2002.

[4] B. M. Ebrahimi and J. Faiz, "Diagnosis and performance analysis of three-phase permanent magnet synchronous motors with static, dynamic and mixed eccentricity," IET Electr. Power Appl., vol. 4, no. 1, pp. 5366, Jan. 2010.

[5] K. Wang, "Phase information at tooth mesh frequency for gear crack diagnosis," in Proceedings of the 2nd IEEE Conference on Industrial Electronics and Applications (ICIEA 2007), Harbin, China, May 23-25 2007, pp. 2712-2717.

[6] C. P. Bottura, J. L. Silvino, and P. de Resende, "A flux observer for induction machines based on a time-variant discrete model," IEEE Trans. Ind. Appl., vol. 29, no. 2, pp. 349-354, Mar./Apr. 1993.
[7] P. L. Jansen and R. D. Lorenz, "A physically insightful approach to the design and accuracy assessment of flux observers for field oriented induction machine drives," IEEE Trans. Ind. Appl., vol. 30, no. 1, pp. 101-110, Jan./Feb. 1994

[8] J. Stephan, M. Bodson, and J. Chiasson, "Real-time estimation of the parameters and fluxes of induction motors," IEEE Trans. Ind. Appl., vol. 30, no. 3, pp. 746-759, May/Jun. 1994.

[9] D.-W. Chung and S.-K. Sul, "Analysis and compensation of current measurement error in vector-controlled AC motor drives," IEEE Trans. Ind. Appl., vol. 34, no. 2, pp. 340-345, Mar./Apr. 1998.

[10] T. G. Habetler, F. Profumo, G. Griva, M. Pastorelli, and A. Bettini, "Stator resistance tuning in a stator-flux field-oriented drive using an instantaneous hybrid flux estimator," IEEE Trans. Power Electron., vol. 13, no. 1, pp. 125-133, Jan. 1998.

[11] H. Kubota, Y. Kataoka, H. Ohta, and K. Matsuse, "Sensorless vector controlled induction machine drives with fast stator voltage offset compensation," in Conference Record of the 34th IEEE Industrial Application Society Annual Meeting, vol. 4, Phoenix, Arizona, USA, Oct. 3-7 1999, pp. 2321-2324.

[12] M. Rodič and K. Jezernik, "An analysis of speed sensorless torque and flux controller for induction motor," in Proceedings of the Power Eelectronics Specialist Conference (PESC), vol. 2, Galway, Ireland, Jun. 18-23 2000, pp. 867-872.

[13] H.-S. Jung, S.-H. Hwang, J.-M. Kim, C.-U. Kim, and C. Choi, "Diminution of current-measurement error for vector-controlled AC motor drives," IEEE Trans. Ind. Appl., vol. 42, no. 5, pp. 1249-1256, Sep./Oct. 2006.

[14] R. Bojoi, P. Guglielmi, and G.-M. Pellegrino, "Sensorless direct fieldoriented control of three-phase induction motor drives for low-cost applications," IEEE Trans. Ind. Appl., vol. 44, no. 2, pp. 475-481, Mar./Apr. 2008.

[15] B. K. Bose and N. R. Patel, "A programmable cascaded low-pass filterbased flux synthesis for a stator flux-oriented vector-controlled induction motor drive," IEEE Trans. Ind. Electron., vol. 44, no. 1, pp. 140-143, Feb. 1997.

[16] J. Hu and B. Wu, "New integration algorithms for estimating motor flux over a wide speed range," IEEE Trans. Power Electron., vol. 13, no. 5 , pp. 969-977, Sep. 1998.

[17] M.-H. Shin, D.-S. Hyun, S.-B. Cho, and S. Choe, "An improved stator flux estimation for speed sensorless stator flux orientation control of induction motors," IEEE Trans. Power Electron., vol. 15, no. 2, pp 312-318, Mar. 2000.

[18] M. Hinkkanen and J. Luomi, "Modified integrator for voltage model flux estimation of induction motors," IEEE Trans. Ind. Electron., vol. 50 no. 4, pp. 818-820, Aug. 2003.

[19] J. Holtz and J. Quan, "Drift- and parameter-compensated flux estimator for persistent zero-stator-frequency operation of sensorless-controlled induction motors," IEEE Trans. Ind. Appl., vol. 39, no. 4, pp. 1052 1060, Jul./Aug. 2003.

[20] M. Zerbo, P. Sicard, and A. Ba-Razzouk, "Accurate adaptive integration algorithms for induction machine drive over a wide speed range," in Proceedings of the IEEE International Conference on Electric Machines and Drives (IEMDC), San Antonio, TX, USA, May 15-18 2005, pp. 1082-1088.

[21] M. Comanescu and L. Xu, "An improved flux observer based on PLL frequency estimator for sensorless vector control of induction motors," IEEE Trans. Ind. Electron., vol. 53, no. 1, pp. 50-56, Feb. 2006.

[22] D. S. L. Dolan and P. W. Lehn, "Simulation model of wind turbine 3p torque oscillations due to wind shear and tower shadow," IEEE Trans. Energy Convers., vol. 21, no. 3, pp. 717-724, Sep. 2006

[23] J. D. Grunnet, M. Soltani, T. Knudsen, M. Kragelund, and T. Bak "Aeolus toolbox for dynamic wind farm model, simulation and control," in Proceedings of the European Wind Energy Conference and Exhibition (EWEC) 2010, Warsaw, Poland, Apr. 20-23 2005.

[24] T. Thiringer and J.-Å. Dahlberg, "Periodic pulsations from a three-bladed wind turbine," IEEE Trans. Energy Convers., vol. 16, no. 2, pp. 128-133, Jun. 2001.

[25] J. Sopanena, V. Ruuskanen, J. Nerg, and J. Pyrhönen, "Dynamic torque analysis of a wind turbine drive train including a direct-driven permanent-magnet generator," IEEE Trans. Ind. Electron., vol. 58, no. 9 pp. 3859-3867, Sep. 2011.

[26] S. Boyd, "Multitone signals with low crest factor," IEEE Trans. Circuits Syst., vol. CAS-33, no. 10, pp. 1018-1022, Oct. 1986. 